increases the risk of developing multiple autoimmune and connective tissue diseases. ${ }^{1}$ Ptpn22 is a negative regulator of Syk and Src family kinases downstream of immuno-receptor signalling cascades. ${ }^{2}$ Fungal $\beta$-glucan receptor dectin-1, signals via Syk kinase, and induces dendritic cells to secrete proinflammatory cytokines IL-1 $\beta, \mathrm{IL}-6, \mathrm{IL}-12 / 23 p 40$ and $\mathrm{TNF} \alpha$, in turn allowing the induction of IL-17 secreting T-cell responses, which are critical to the clearance of fungal infections. ${ }^{3} \mathrm{IL}-17$ has been implicated as a key cytokine in inflammatory responses associated with RA, JIA, and psoriasis. ${ }^{4}$

Objectives: To investigate if Ptpn22 regulates dectin-1 signalling and controls the capability of dectin-1 matured BMDC to promote adaptive immune responses. Methods: GM-CSF bone marrow derived dendritic cells (BMDC) were generated from C57BL/6 WT, Ptpn22-- or Ptpn22 ${ }^{\mathrm{R} 619 \mathrm{~W}}$ (human PTPN22 ${ }^{\mathrm{R} 620 \mathrm{~W}}$ orthologue) mice, and pulsed with $\mathrm{OVA}_{323-339}$ in the presence or absence of the dectin-1 agonist curdlan. Activated BMDC were co-cultured in vitro with OT-II T-cells or adoptively transferred into OT-II mice and the resulting T-cell response assessed. Cytokine secretion from curdlan activated Ptpn22 variant mouse BMDC was determined by immunoassay and the kinetics of Syk and Erk phosphorylation were determined by immunoblot.

Results: We observed that Dectin-1 activated Ptpn22-- BMDC had an enhanced capability to induce T-cell IL-17 secretion both in vitro and in vivo compared to WT BMDC. Following dectin-1 priming Ptpn22 ${ }^{-/}$BMDC secreted increased IL-1 $\beta$ compared to WT BMDC, and the increase in IL-1 $\beta$ was found to be sufficient to cause the enhanced IL-17 response induced by Ptpn22 $2^{-1}$ BMDC. Dectin- 1 induced IL-1 $\beta$ secretion was found to be Syk and Erk dependent and assessment of Syk and Erk kinetics of phosphorylation revealed that dectin-1 activated Ptpn22-1BMDC displayed enhanced Syk and Erk phosphorylation compared to WT BMDC. Furthermore, Ptpn22 ${ }^{\mathrm{R} 619 \mathrm{~W}}$ BMDC (orthologue of human Ptpn22 ${ }^{\mathrm{R} 620 \mathrm{~W}}$ ) exhibited a similar enhancement in IL-1 $\beta$ secretion and induced enhanced T-cell dependent IL-17 responses in vivo, indicating that the PTPN22 polymorphism behaves as a loss-of-function allele in the context of dectin-1 signals.

Conclusions: Data highlight Ptpn22 as a novel regulator of dectin-1 signals and provide a link between genetically conferred perturbation to innate receptor signalling pathways and autoimmunity.

References:

[1] Burn, G. L., Svensson, L., Sanchez-Blanco, C., Saini, M. \& Cope, A. P. Why is PTPN22 a good candidate susceptibility gene for autoimmune disease? FEBS Lett. 585, 3689-98 (2011).

[2] Cloutier, J. F. \& Veillette, A. Cooperative inhibition of T-cell antigen receptor signaling by a complex between a kinase and a phosphatase. J. Exp. Med. 189, 111-21 (1999).

[3] Dambuza, I. M. \& Brown, G. D. C-type lectins in immunity: recent developments. Curr. Opin. Immunol. 32, 21-27 (2015).

[4] Tabarkiewicz, J., Pogoda, K., Karczmarczyk, A., Pozarowski, P. \& Giannopoulos, K. The Role of IL-17 and Th17 Lymphocytes in Autoimmune Diseases. Arch. Immunol. Ther. Exp. (Warsz). 63, 435-49 (2015).

Disclosure of Interest: None declared

DOI: 10.1136/annrheumdis-2017-eular.4717

\section{OP0297 FUNCTIONAL POLYMORPHISMS OF THE ABCG2 GENE IN PRIMARY GOUT}

B. Stiburkova ${ }^{1,2}$, K. Pavelcova ${ }^{3,4}$, L. Petru ${ }^{3,4}$, P. Cepek ${ }^{3}$, J. Zavada ${ }^{3}$, K. Pavelka ${ }^{3}{ }^{1}$ Institute of Rheumatology, Prague $2 ;{ }^{2}$ Institute of Inherited Metabolic Disorders, First Faculty of Medicine, Charles University; ${ }^{3}$ Institute of Rheumatology; ${ }^{4}$ Department of Rheumatology, First Faculty of Medicine, Charles University, Prague, Czech Republic

Background: The urate transporters are one of the genetic determinants of serum uric acid concentrations. Common dysfunctional variants of $A B C G 2$ are revealed to be a major cause of gout and hyperuricemia by decreasing urate excretion. In this study we describe the analysis of allelic variants in the ABCG2 in a cohort with primary gout.

Objectives: The cohort was recruited in the Institute of Rheumatology, Prague. Gouty arthritis was diagnosed in 146 subjects (132 men, 14 women) according to the American college of rheumatology criteria 1977. Patients suffering from secondary gout and other purine metabolic disorders associated with pathological concentrations of serum uric acid were excluded.

Methods: In each patient, the family history of gout, number of gouty attacks, the presence of tophi, the occurrence of associated diseases and therapy have been assessed. All 16 exons of ABCG2 were amplified using PCR, and sequenced directly.

Results: In the $A B C G 2$ gene, 19 intronic sequence variants were detected. In the case of $c \cdot 689+1 \mathrm{G}>\mathrm{A}$, related to an individual with severe gouty phenotype, two abnormal splicing variants were identified: a) r.[532_689del]; b) r.[532 689del], r.[944 949del]. Identified deletions lead to frameshift and premature stop codon introduction ${ }^{1}$. From the 12 exon variants detected, there were nine non-synonymous: p.V12M, p.Q141K, p.R147W, p.T153M, p.F373C, p.T421A, p.T434M, p.S476P and p.D620N. Heterozygous p.V12M was detected in seven individuals. Heterozygous variants p.R147W, p.T153M, p.F373C, p.T421A, p.T434M and p.S476P were detected once, variant p.D620N twice. All these allelic variants were in silico predicted as a probably damaging. The p.Q141K, previously functionally characterized allelic variant with a strong effect on uric acid secretion impairment, was in cohort of gout patients presented with significantly higher minor allele frequency 0.24 (MAF), 57 heterozygotes/6 homozygotes, than in population of European origin $(\mathrm{MAF}=0.09)$ and world-wide population (MAF=0.12). In our cohort, the age of onset of gout had a normal distribution. Remarkably, in $75 \%$ of all individuals with early onset of gout between age 10-20 years, we detected allelic variant p.Q141K (in 6 out of 8 patients). In the age group $21-30$ years, this allelic variant was detected in $42 \%$ of patients (8/19), and in the age group $61-70$ years, just in $29 \%$ (13/29).

Conclusions: Our results show that genetic factor $A B C G 2$ should be considered as one of the strong common risks for gout. In summary, we revealed that allelic variants of $A B C G 2$, especially dysfunctional variant p.Q141K, have a significant effect on earlier onset of gout.

\section{References:}

[1] Stiburkova B et al. Novel dysfunctional variant in ABCG2 as a cause of severe tophaceous gout: biochemical, molecular genetics and functional analysis. Rheumatology (Oxford). 2016 Jan;55(1):191-4.

Acknowledgements: This study was supported by the grants from the Czech Republic Ministry of Health AZV 15-26693A.

Disclosure of Interest: None declared

DOI: 10.1136/annrheumdis-2017-eular.4901

\section{OP0298 CHROMATIN INTERACTIONS REVEAL NOVEL GENE TARGETS FOR DRUG REPOSITIONING IN RHEUMATIC DISEASES}

P. Martin ${ }^{1}$, K. Duffus ${ }^{1}$, A. McGovern ${ }^{1}$, A. Yarwood ${ }^{1}$, A. Barton ${ }^{1,2}$ J. Worthington ${ }^{1,2}$, S. Eyre ${ }^{1}$, G. Orozco ${ }^{1} .{ }^{1}$ Arthritis Research UK Centre for Genetics and Genomics, University of Manchester; ${ }^{2}$ NIHR Manchester Musculoskeletal Biomedical Research Unit, Central Manchester Foundation Trust, Manchester Academic Health Science Centre, Manchester, United Kingdom

Background: The treatment of rheumatic diseases can be both expensive and ineffective with up to $1 / 3$ of patient's failing to respond to current treatments. There is therefore a need to identify new treatments and to target these to individual patients. Although genetic studies have been successful in identifying common variation associated with disease susceptibility, a large proportion of these lie outside protein-coding regions. Many show enhancer activity but it is often unclear which gene(s) they regulate and how they contribute to disease. Chromatin folding brings linearly distant areas of the genome, such as promoters and enhancers, into close proximity, driving gene expression. Capture $\mathrm{Hi}-\mathrm{C}(\mathrm{CHi}-\mathrm{C})$ interrogates these interactions in a high-throughput, high-resolution manner, linking implicated enhancers to causal genes.

Objectives: Utilising our existing $\mathrm{CHi}-\mathrm{C}$ data on 3 rheumatic diseases, RA, JIA and PsA, targeting all known genetic associations, we explored the potential to identify candidate causal genes that are targets for existing drugs, which could be repositioned for use in these diseases.

Methods: Chromatin interaction data for $\mathrm{T}-$ and $\mathrm{B}$-cells in the 3 diseases was analysed using CHiCAGO v2 using a score cut-off of $\geq 5$. Interactions between disease regions and promoters were identified using BEDTOOLS v2.21.0 and intersected with drug targets from DrugBank v4.5.0. Existing treatments for each disease were identified by the presence of the relevant name in the "indication" field.

Results: Overall 850 genes were identified as interacting with a disease associated region. Of these, 61 are existing drug targets (303 drugs) (Table 1) and 9 are existing therapies used in the treatment of disease, primarily RA.

\begin{tabular}{lcccccc}
\multicolumn{6}{l}{ Table 1. Summary of drug targets identified by CHi-C } \\
\hline Disease & $\begin{array}{c}\text { Number of } \\
\text { identified } \\
\text { by CHi-C }\end{array}$ & $\begin{array}{c}\text { Number of } \\
\text { genes which } \\
\text { are existing } \\
\text { drug targets }\end{array}$ & $\begin{array}{c}\text { Number of } \\
\text { drugs } \\
\text { identified }\end{array}$ & $\begin{array}{c}\text { Number of } \\
\text { drugs } \\
\text { withdrawn }\end{array}$ & $\begin{array}{c}\text { Number of } \\
\text { drugs currently } \\
\text { used }\end{array}$ & $\begin{array}{c}\text { Number of drugs } \\
\text { for potential } \\
\text { repositioning }\end{array}$ \\
\hline RA & 510 & 41 & 106 & 4 & 9 & 93 \\
JIA & 324 & 16 & 74 & 0 & 1 & 73 \\
PSA & 196 & 13 & 147 & 0 & 0 & 147 \\
All & 850 & 61 & 303 & 4 & 9 & 290 \\
\hline
\end{tabular}

Conclusions: Our study identifies genes which are implicated in disease, are the target of existing drugs and offer the potential for drug repositioning. Of the potential drugs identified for RA, 14 are used in the treatment of various cancers and 11 are used in the treatment of diabetes and multiple sclerosis. Interestingly, 17 potential drugs identified for PsA are used in the treatment of schizophrenia and 11 in the treatment of hypertension. This data shows a novel insight into how functional annotation of genetic associations in rheumatic diseases can provide gene targets for re-positioned therapies.

Acknowledgements: This work was funded by Wellcome Trust Research Career Development Fellowship (095684); Arthritis Research UK (grant numbers 20385, 20571); Wellcome Trust (097820/Z/11/B); European Union's FP7 Health Programme (FP7-HEALTH-F2-2012-305549, Euro-TEAM, FP7/2007-2013); Innovative Medicines Initiative (BeTheCure project 115142); Medical Research Council (MR/K015346/1); National Institute for Health Research Manchester Musculoskeletal Biomedical Research Unit; Biotechnology and Biological Sciences Research Council UK (BBS/E/B/000C0405).

Disclosure of Interest: None declared

DOI: 10.1136/annrheumdis-2017-eular.2685 